Article

\title{
Countermovement Jump Analysis Using Different Portable Devices: Implications for Field Testing
}

\author{
Vincenzo Rago ${ }^{1,2, *}$, João Brito ${ }^{2}$, Pedro Figueiredo ${ }^{2,3}$, Thiago Carvalho ${ }^{1}$, Tiago Fernandes ${ }^{1}$ (D), \\ Pedro Fonseca ${ }^{4}$ and António Rebelo ${ }^{1}$ \\ 1 Center of Research, Education, Innovation and Intervention in Sports, University of Porto, 4099-002 Porto, \\ Portugal; up201005205@fade.up.pt (T.C.); 201301434@fade.up.pt (T.F.); anatal@fade.up.pt (A.R.) \\ 2 Portugal Football School, Portuguese Football Federation, 1495-433 Oeiras, Portugal; joao.brito@fpf.pt (J.B.); \\ pedro.figueiredo@fpf.pt (P.F.) \\ 3 Research Center in Sports Sciences, Health Sciences and Human Development, CIDESD, University Institute \\ of Maia, ISMAI, 4475-690 Maia, Portugal \\ 4 Porto Biomechanics Laboratory, University of Porto, 4099-002 Porto, Portugal; pedro.labiomep@fade.up.pt \\ * Correspondence: Vincenzo.rago@fpf.pt; Tel.: +351-96-106-7495
}

Received: 23 August 2018; Accepted: 30 August 2018; Published: 31 August 2018

check for updates

\begin{abstract}
The aim of this study was to analyze the concurrent validity, test-retest reliability, and capacity to detect changes of four different portable devices used to measure a wide range of neuromuscular parameters derived from countermovement jump (CMJ). An accelerometric device (Myotest), a jump mat (Ergojump), an optical device (Optojump), and a smartphone app (MyJump) were simultaneously examined for concurrent validity against gold-standard measures (motion-capture system and a force platform). Twenty-two CMJ-derived variables were collected from 15 healthy male subjects $(n=60 \mathrm{CMJs})$. Contraction time (CT) and eccentric duration (EccD) measurements obtained from the Myotest were moderately to largely associated with and not different from force platform measurements ( $\mathrm{r}=0.31$ to 0.64 , ES $=0.11$ to 0.18 ) and showed moderate test-retest reliability (intraclass correlation coefficient $(\mathrm{ICC})=0.92$ to 0.97 , coefficient of variation $(\mathrm{CV})=3.8$ to $8.0 \%$ ). Flight time (FT) and jump height (JH) from Ergojump, Optojump, and MyJump showed moderate to strong associations with gold-standard measurements $(\mathrm{r}=0.57$ to 0.98$)$ and good test-retest reliability (ICC $=0.54$ to $0.97, \mathrm{CV}=1.8$ to 4.2 ). However, all portable devices underestimated $\mathrm{JH}(\mathrm{ES}=1.25$ to 2.75$)$. Independent of the instrument used, the analyzed CMJ variables showed good capacity to detect changes (standard error of measurement (SEM) < smallest worthwhile change $(\mathrm{SWC})$ ), with the exception of rate of force and rate of power development parameters, which showed marginal capacity $(\mathrm{SEM}>\mathrm{SWC}$ ). The Myotest is preferable to measure temporal parameters during ground contact, whereas Ergojump, Optojump, and MyJump devices may be preferable to measure FT and JH, with the Optojump being the most accurate.
\end{abstract}

Keywords: force platform; motion analysis; neuromuscular assessment; measurements; reliability; validity

\section{Introduction}

The countermovement jump (CMJ) is one of the most popular tests to monitor an athlete's muscle power of the lower extremities. An athlete's CMJ performance is relevant in a variety of sports and commonly quantified by jump height (JH) or flight time (FT), which have been considered as indicators of vertical jump performance [1,2] and used to measure training adaptations and monitor neuromuscular fatigue [3]. However, CMJ execution is dependent on various time and force components throughout the various phases preceding the flight [4]. Therefore, the use of $\mathrm{JH}$ or 
FT in isolation as a global indicator of muscle power in the lower extremities does not inform about the specific contribution of neuromuscular components during ground contact, consequently masking training-induced adaptations or neuromuscular fatigue.

CMJ performance variables have been adopted to describe chronic neuromuscular adaptations and acute neuromuscular fatigue in response to exercise, but contradictory results have been observed. Silva et al. [2] examined the correlation between individual match exposure and changes in JH in professional athletes throughout an entire season, reporting no significant training-induced effects. On the other hand, reports in young athletes showed negative associations between various training load parameters (e.g., session rating of perceived exertion exposure time to training) and changes in $\mathrm{JH}$ [5]. In addition, $\mathrm{JH}$ significantly dropped in response to recreational $5 \mathrm{v} 5$ small-sided soccer games [6], whereas CMJ height was not altered following $4 \mathrm{v} 4$ and $8 \mathrm{v} 8$ small-sided soccer games [7]. Taken together, these findings indicate that JH or FT per se does not elucidate an eventual mask over acute fatigue responses or over chronic adaptations to exercise. In this context, critical information can be directly extracted from the force-time curves during the CMJ, and various parameters (e.g., contraction duration, time to peak force/power) have been recently deemed suitable for neuromuscular fatigue detection. Regarding neuromuscular adaptations, variations in force-time parameters (e.g., flight time to contraction time ratio) were observed in Australian football players over the course of a season, indicating sensitivity to increases with training load over time [8]. Moreover, during a six-week training block with progressive increases in training load, subjective wellness was positively associated with changes in maximum rate of force development, force at zero velocity, and FT $(r=0.28-0.34)$ in female rugby players [9].

In laboratory conditions, $\mathrm{CMJ}$ time and force components may be accurately assessed, providing valid and reliable results using a force platform (FP) $[10,11]$ or video-based motion capture system (MCAP) [1]. For instance, Gathercole et al. [12] examined the reliability and fatigue sensitivity of the $\mathrm{CMJ}$ test using an FP, reporting a meaningful reduction in 18 neuromuscular variables obtained from $\mathrm{CMJ}$ after a high-intensity fatiguing protocol. These findings suggest that acute fatigue can induce a decrease of peak force and eccentric function and an increase of jump duration [10]. Although FP and MCAP are considered gold standards for CMJ measurement, limited access and associated costs make them unsuitable, especially for field-based testing. Therefore, it would be of interest to understand whether portable equipment frequently used in CMJ testing would allow the assessment of valid and reliable time and force components of CMJ. For example, the Myotest device uses a body accelerometer and has been shown to be a valid and reliable method for the assessment of JH $[13,14]$. Moreover, the traditional switch mat consists of a digital apparatus connected by a cable to a resistive platform, and can be used on flat surface to test CMJ [15]. Optical systems have been proposed to determine JH by measuring FT [16]. Even more recently, promising results were found from an iPhone app (MyJump), showing excellent validity in relation to FP to measure JH based on the flight-time method [17].

No studies have attempted to simultaneously analyze the suitability of a wide range of portable equipment to characterize athletes' neuromuscular performance. The purpose of this study was to analyze the concurrent validity, test-retest reliability, and capacity to detect changes of four different portable devices used to measure a wide range of CMJ-derived neuromuscular parameters.

\section{Materials and Methods}

\subsection{Subjects}

Fifteen male undergraduate sports sciences students with an average age of $27.0 \pm 5.0$ years, height of $174.0 \pm 9.0 \mathrm{~cm}$, body mass of $72.2 \pm 8.28 \mathrm{~kg}$, and training hours of $5.0 \pm 2.0 \mathrm{~h}$ per week were tested in January 2018. They were regularly involved in various activities such as weight lifting, resistance training, and recreational team sports, and had been injury-free during the last 2 years. All volunteers agreed to provide their maximum effort during all laboratory tests, and they were formally and verbally informed that they were free to withdraw from the study without any 
consequences. The ethical board of the Faculty of Sports, University of Porto approved and recorded the study under CEFADE.08.2018.

\subsection{Experimental Design}

Four portable devices were examined against the gold standard (FP and MCAP) using 5 CMJs [15] performed by each participant. Each CMJ for each piece of equipment was entered in the database as a single case for intratrial assessment. This methodological approach was used to eliminate the intertrial variability provided by single jump condition performance (i.e., comparisons between independent jump conditions) $[13,18]$. The independent variable was the device, whereas 22 CMJ-derived parameters, listed in Table 1, were set as the dependent variables.

Table 1. Definitions of neuromuscular parameters adopted.

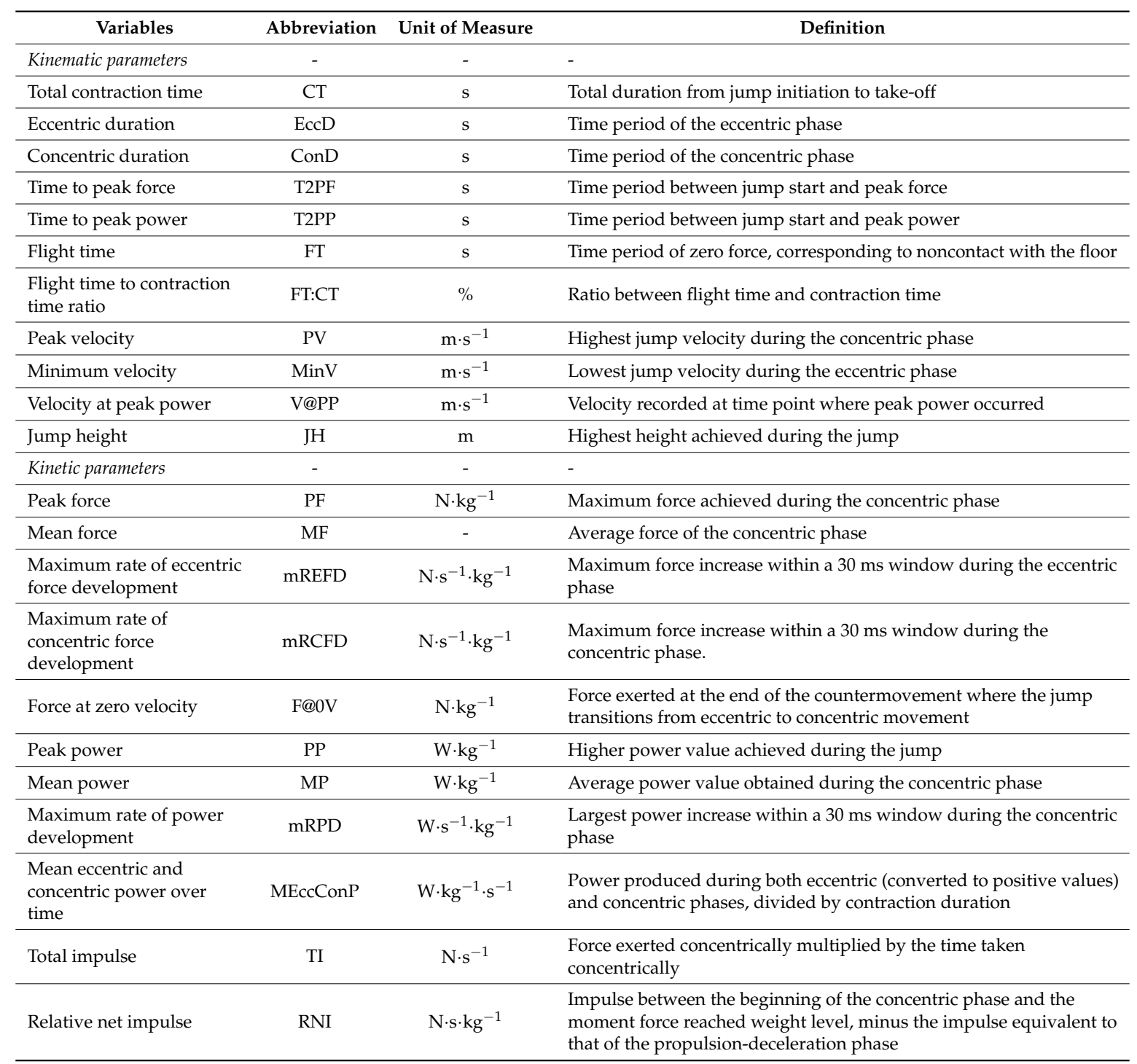

Despite the various vertical jump protocols that have been proposed $[7,14], \mathrm{CMJ}$ has been considered the most popular and easiest to perform [13]. The subjects received instructions about how to correctly perform CMJ in a separate familiarization session $24 \mathrm{~h}$ before data collection. On the data collection day, each subject performed a 5 min cycling warmup on a stationary bike and 2 preliminary CMJs. Then, equipped with an accelerometric system, they were positioned in an upright stance with their feet shoulder-width apart and their toes pointed forward or slightly outward, on the center of 
the FP, on a switch mat, within optical equipment with light-emitting diodes (LEDs), and in front of a smartphone, simultaneously. The participants executed the jump by flexing their knees to a position they perceived to be comfortable (i.e., preferred starting push-off position) [11,19]. To limit possible variations in posture during the jump that could affect the final assessment, the no-arm swing version was used [13]. At least $30 \mathrm{~s}$ recovery was allowed between jump trials.

Eleven kinematic parameters suggested by Gathercole et al. [12] and 11 kinetic parameters previously described elsewhere $[4,12,20]$ were considered (Table 1 ). All kinetic parameters were scaled to the subject's body mass.

\subsection{Procedures}

\subsubsection{Gold-Standard Measurements}

A motion capture system (Qualisys AB, Göteborg, Sweden) operating at a $200 \mathrm{~Hz}$ sampling frequency was used. Because CMJ is mainly executed in the sagittal plane, a marker was attached to the left lateral femoral condyle to avoid recording displacement in the frontal plane associated with trunk flexion. This also allowed the mark to be placed as close to the center of mass as possible, enabling tracking along all CMJ phases [19]. This system is capable of ignoring other movement, considering only vertical displacement. The marker displacement, peak, and minimum velocity from the starting position to peak JH were quantified. Second, an extensiometric FP (Bertec FP9060, Bertec, Columbus, KY, USA) embedded in the floor, with a sampling frequency of $2000 \mathrm{~Hz}$, was used. Motion capture and FP data were simultaneously collected using Qualisys Track Manager software (Qualisys $\mathrm{AB}$, Göteborg, Sweden). Force platform variables were computed from the force-velocity, -time, and -acceleration traces using a custom-made MATLAB routine (MATLAB, MathWorks, Natick, MA, USA). According to a specific variable, only 1 criterion (MCAP or FP) was chosen as gold standard.

\subsubsection{Accelerometric System}

The Myotest Pro (Myotest, Sion, Switzerland) uses a 3-dimensional accelerometer inserted into a small box (dimensions $5.5 \times 8.5 \times 2.3 \mathrm{~cm}$, mass $50 \mathrm{~g}$ ) that is secured at waist level with a purpose-built strap (Velcro belt) with an elastic waistband. The Myotest measures acceleration on the vertical axis of the load, on which the accelerometer sensor is fixed, using a sampling rate of $200 \mathrm{~Hz}$. The device was perpendicularly attached to a large $(8.5 \mathrm{~cm})$ Velcro elastic belt provided with the device. This was fixed to hip level on the left side of the body, as indicated by the manufacturer, and attached to the left lateral femoral head to reduce the possible accelerations induced by trunk flexion (during the eccentric phase) and extension (i.e., propulsion), thus emphasizing vertical displacement. Accelerometry data were stored during the assessments and subsequently downloaded using the proprietary software (Myotest PRO Software version 1.0, Myotest, Sion, Switzerland), and further neuromuscular variables were computed from raw data of acceleration over time, using a custom-made MATLAB routine (MATLAB, MathWorks, Natick, MA, USA). For the accelerometric system, JH was computed using the flight-time method [10].

\subsubsection{Switch Mat}

The Ergojump mat recorded FT from take-off to landing. The switch mat was placed on top of the floor-embedded force platform and the mat's weight was removed from the force platform data. This equipment provides JH and FT immediately following the jump. Furthermore, peak power was also calculated using the Sayers equation [21] as follows:

$$
\text { Peak power }(\mathrm{W})=61.9 \times \mathrm{JH}(\mathrm{cm})+36.0 \times \text { subject's body mass }(\mathrm{kg})+1.822
$$

\subsubsection{Optical Equipment with Light-Emitting Diodes}

The Optojump system (Optojump, Microgate, Bolzano, Italy) consists of 2 bars (transmitting and receiving bars, $1 \mathrm{~m}$ apart) equipped with 33 optical LEDs fitted in the transmitting bar that continuously 
communicate with the corresponding set in the receiving bar. The LEDs are positioned $0.3 \mathrm{~cm}$ from ground level, on the Ergojump mat, and at $3.125 \mathrm{~cm}$ intervals. Any break of the beam switched on and off automatically activates a digital chronometer used to calculate FT and JH [10].

\subsubsection{Smartphone App}

The MyJump app was installed on an iPhone 6, which includes a $120 \mathrm{~Hz}$ high-speed camera, at a quality of $720 \mathrm{p}$. MyJump was designed to calculate the time (in ms) between 2 frames selected by the user and subsequently $\mathrm{JH}$ using the following equation:

$$
\mathrm{JH}=\mathrm{FT}^{2} \times 1.22625
$$

A researcher lay prone on the ground with the iPhone 6 facing the participant (in the frontal plane) $\sim 1.5 \mathrm{~m}$ from the force platform at floor level, and zoomed in on the feet of the participant. The MyJump app provides JH, FT, and peak velocity. Peak power was also computed as previously described.

\subsection{Statistical Analysis}

In accordance with the outlier removal procedure suggested by Gathercole et al. [12], we selected the 4 out of 5 most consistent CMJ repetitions (differing least from the mean) based on mean eccentric and concentric power over time. Therefore, only 4 CMJs collected were considered for analysis, resulting in 60 cases across the 75 jumps performed. All variables were normally distributed, and data are reported as mean \pm standard deviation (SD).

Correlations between the gold standard and portable devices were quantified using the following criteria: $\leq 0.1$ (trivial), $0.1-0.3$ (small), 0.3-0.5 (moderate), 0.5-0.7 (large), 0.7-0.9 (very large), and $\geq 0.9$ (almost perfect). Substantial over- and underestimations were analyzed using paired sample $t$-test. Differences were interpreted using effect size (ES) according to Hopkins [22] as trivial $(<0.2)$, small (0.2-0.6), moderate (0.6-1.2), large (1.2-2.0), very large (2.0-4.0), and huge ( $>4.0)$. When $90 \%$ confidence intervals (CIs) overlapped positive and negative values, the effect was deemed as unclear. Otherwise, the magnitude is reported as the observed value. A large correlation $(r \geq 0.90)$ and a trivial difference $(\mathrm{ES}<0.2)$ indicated sufficient validity. Test-retest reliability was assessed using intraclass correlation coefficient (ICC) (absolute reliability [1,2], a 2-way random effects model with single measure) and coefficients of variation (CV; relative reliability). For ICC, we used criteria suggested by Portney and Watkins [23] to evaluate reliability as follows: $\geq 0.75$ (good), 0.50-0.74 (moderate), $0.26-0.50$ (fair), and $\leq 0.25$ (poor). $C V$ was calculated by dividing the within-subjects SD by the mean and then multiplying by 100 , qualitatively classified as good $(<5 \%)$, moderate $(5-10 \%)$, and poor ( $>10 \%)$. ICC $\geq 0.75$ and CV $\leq 10 \%$ were defined as sufficient reliability. Standard error of measurement (SEM) was calculated by dividing the SD of the difference score between test and retest by $\sqrt{\text { ICC }}$ [24]. The smallest worthwhile change (SWC) was determined to establish the usefulness of the test by multiplying the between-subject standard deviation by 0.2. If SEM is smaller than SWC, the ability to detect a change is "good"; if SEM equals SWC, then the test is "satisfactory"; but if SEM is greater than SWC, then the test is rated as "marginal" [25]. The minimal detectable change ( $\left.\mathrm{MDC}_{95}\right)$ was calculated as $\mathrm{MDC}_{95}=\mathrm{SEM} \times \sqrt{2} \times 1.96$ [25].

\section{Results}

Descriptive values are reported in Table 2. Differences and correlations between gold standard and portable devices are reported in Figures 1 and 2, respectively. 
Table 2. Descriptive values of countermovement jump (CMJ) kinematic variables obtained with different devices. Values are mean \pm standard deviation (SD).

\begin{tabular}{|c|c|c|c|c|c|c|}
\hline Variable & MCAP & Force Platform & Myotest & Ergojump & Optojump & MyJump \\
\hline \multicolumn{7}{|c|}{ Kinematic parameters } \\
\hline CT (s) & - & $0.77 \pm 0.16$ & $0.76 \pm 0.09$ & - & - & - \\
\hline EccD (s) & - & $0.50 \pm 0.15$ & $0.50 \pm 0.08$ & - & - & - \\
\hline ConD (s) & - & $0.26 \pm 0.02$ & $0.26 \pm 0.04$ & - & - & - \\
\hline $\mathrm{T} 2 \mathrm{PF}(\mathrm{s})$ & - & $0.55 \pm 0.17$ & $0.60 \pm 0.10$ & - & - & - \\
\hline T2PP (s) & - & $0.71 \pm 0.16$ & $0.67 \pm 0.09$ & - & - & - \\
\hline FT (s) & - & $0.54 \pm 0.03$ & $0.49 \pm 0.04$ & $0.55 \pm 0.06$ & $0.53 \pm 0.03$ & $0.57 \pm 0.03$ \\
\hline
\end{tabular}

Table 2. Cont.

\begin{tabular}{|c|c|c|c|c|c|c|}
\hline Variable & MCAP & Force Platform & Myotest & Ergojump & Optojump & MyJump \\
\hline FT:CT (\%) & - & $0.72 \pm 0.16$ & $0.66 \pm 0.10$ & - & - & - \\
\hline $\mathrm{PV}\left(\mathrm{m} \cdot \mathrm{s}^{-1}\right)$ & $3.04 \pm 0.23$ & - & $2.08 \pm 0.39$ & - & - & $1.42 \pm 0.23$ \\
\hline $\operatorname{MinV}\left(\mathrm{m} \cdot \mathrm{s}^{-1}\right)$ & $-1.25 \pm 0.16$ & - & $-1.08 \pm 0.26$ & - & - & - \\
\hline $\mathrm{V} @ P P\left(m \cdot s^{-1}\right)$ & - & $2.47 \pm 0.15$ & $1.89 \pm 0.34$ & - & - & - \\
\hline $\mathrm{JH}(\mathrm{m})$ & $0.46 \pm 0.04$ & - & $0.42 \pm 0.04$ & $0.38 \pm 0.07$ & $0.35 \pm 0.04$ & $0.41 \pm 0.04$ \\
\hline \multicolumn{7}{|c|}{ Kinetic parameters } \\
\hline $\mathrm{PF}\left(\mathrm{N} \cdot \mathrm{kg}^{-1}\right)$ & - & $23.88 \pm 1.99$ & $22.23 \pm 2.37$ & - & - & - \\
\hline $\mathrm{MF}\left(\mathrm{N} \cdot \mathrm{kg}^{-1}\right)$ & - & $13.39 \pm 1.24$ & $11.96 \pm 0.82$ & - & - & - \\
\hline $\operatorname{mREFD}\left(\mathrm{N} \cdot \mathrm{s}^{-1} \cdot \mathrm{kg}^{-1}\right)$ & - & $136.88 \pm 69.88$ & $101.27 \pm 36.22$ & - & - & - \\
\hline $\operatorname{mRCFD}\left(\mathrm{N} \cdot \mathrm{s}^{-1} \cdot \mathrm{kg}^{-1}\right)$ & - & $29.37 \pm 19.93$ & $57.44 \pm 23.82$ & - & - & - \\
\hline $\mathrm{F} @ 0 \mathrm{~V}\left(\mathrm{~N} \cdot \mathrm{kg}^{-1}\right)$ & - & $23.16 \pm 2.31$ & $18.77 \pm 3.30$ & - & - & - \\
\hline $\mathrm{PP}\left(\mathrm{W} \cdot \mathrm{kg}^{-1}\right)$ & - & $36.01 \pm 8.80$ & $47.84 \pm 10.17$ & $46.18 \pm 5.38$ & $51.47 \pm 3.80$ & - \\
\hline $\mathrm{MP}\left(\mathrm{W} \cdot \mathrm{kg}^{-1}\right)$ & - & $28.66 \pm 2.51$ & $18.89 \pm 5.17$ & - & - & - \\
\hline $\operatorname{mRPD}\left(\mathrm{W} \cdot \mathrm{s}^{-1} \cdot \mathrm{kg}^{-1}\right)$ & - & $345.49 \pm 81.79$ & $301.08 \pm 84.41$ & - & - & - \\
\hline $\operatorname{MEccConP}\left(\mathrm{W} \cdot \mathrm{kg}^{-1} \cdot \mathrm{s}^{-1}\right)$ & - & $13.63 \pm 2.29$ & $9.14 \pm 2.04$ & - & - & - \\
\hline TI $\left(\mathrm{N} \cdot \mathrm{s}^{-1}\right)$ & - & $197.00 \pm 29.77$ & $121.74 \pm 47.34$ & - & - & - \\
\hline $\mathrm{RNI}\left(\mathrm{N} \cdot \mathrm{s}^{-1} \cdot \mathrm{kg}^{-1}\right)$ & - & $2.61 \pm 0.16$ & $1.70 \pm 0.40$ & - & - & - \\
\hline
\end{tabular}

MCAP, motion capture system; ConD, concentric duration; CT, total contraction time; EccD, eccentric duration; F@0V, force at zero velocity; FT, flight time; FT:CT, flight time to contraction time ratio; MP, mean power; MEccConP, mean eccentric and concentric power over time; MinV, minimum velocity; mRCFD, maximum rate of concentric force development; mRCED, maximum rate of eccentric force development; $\mathrm{mRPD}$, maximum rate of power development; PF, peak force; PP, peak power; PV, peak velocity; RNI, relative net impulse; T2PF, time to peak force; T2PP, time to peak power; TI, total impulse; V@PP, velocity at peak power. 


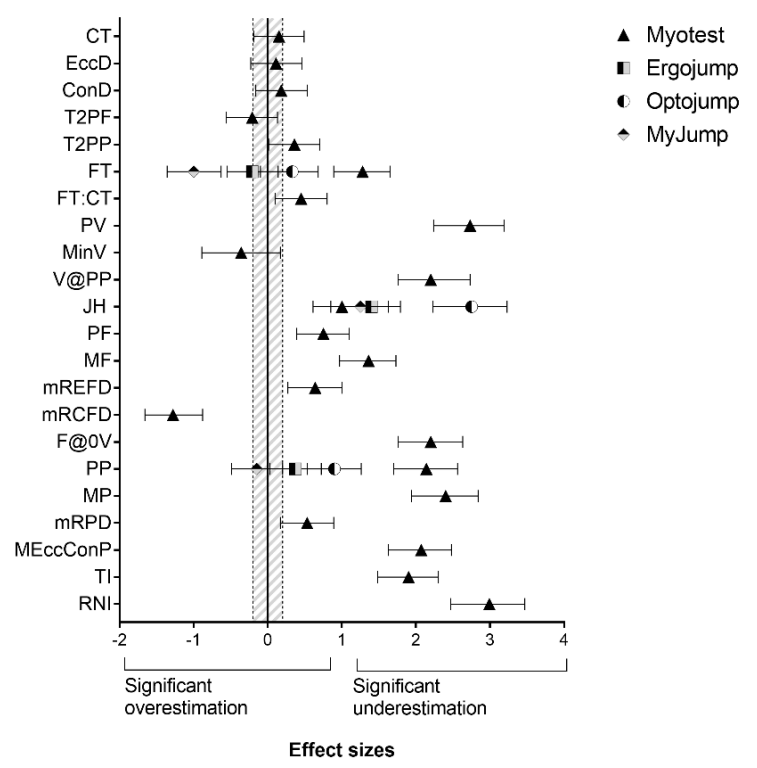

Figure 1. Differences between gold-standard measurements and four portable devices in 22 neuromuscular parameters. Shaded area indicates trivial differences $(\mathrm{ES}<0.2)$. ConD, concentric duration; CT, total contraction time; EccD, Eeccentric duration; ES, effect size; F@0V, force at zero velocity; FT, flight time; FT:CT, flight time to contraction time ratio; ICC, intraclass correlation coefficient; $\mathrm{MF}$, mean force; MP, mean power; MEccConP, mean eccentric and concentric power over time; MinV, minimum velocity; $\mathrm{mRCFD}$, maximum rate of concentric force development; $\mathrm{mREFD}$, maximum rate of eccentric force development; mRPD, maximum rate of power development; PF, peak force; PP, peak power; PV, peak velocity; RNI, relative net impulse; $\mathrm{T} 2 \mathrm{PF}$, time to peak force; $\mathrm{T} 2 \mathrm{PP}$, time to peak power; TI, total impulse; V@PP, velocity at peak power. Gold standard is a motion capture system for PV, $\mathrm{MinV}$, and $\mathrm{JH}$, and force platform for all remaining variables.

$\mathrm{CT}$, EccD, concentric duration (ConD), and peak force (PF) obtained from the Myotest were moderately to largely correlated and not significantly different from FP measurements $(\mathrm{r}=0.31-0.64$, $\mathrm{ES}=0.11-0.18$ ). In addition, $\mathrm{FT}, \mathrm{JH}, \mathrm{PF}$, maximum rate of eccentric force (mREFD), maximum rate of concentric force development (mRCFD), force at zero velocity (F@0V), maximum rate of power development (mRPD), and time to peak power (TI) measurements were positively correlated with but underestimated FP measurements $(\mathrm{r}=0.44-0.71, \mathrm{ES}=0.53-2.20)$. The remaining variables were not valid when compared to gold-standard measurements. FT obtained from Ergojump was positively correlated with and not different from FP $(r=0.61[0.09 ; 0.32]$, ES $=0.21[-0.55 ; 0.14])$. In contrast, $\mathrm{JH}$ was strongly associated with but substantially different from FP $(\mathrm{r}=0.69 ; \mathrm{ES}=1.40$ [1.00; 1.79]). FT obtained from Optojump was valid in relation to FP $(\mathrm{r}=0.98[0.97 ; 0.99]$, ES $=0.33[-0.01 ; 0.68])$. Despite JH from Optojump being almost perfectly correlated with these devices, they were significantly different $(\mathrm{r}=0.90 ; \mathrm{ES}=2.75$ [2.23; 3.23]). FT and JH obtained from MyJump were strongly associated with FP and MCAP $(r=0.67$ [0.54; 0.67] and 0.66 [0.53; 0.76], respectively). However, FT overestimated $\mathrm{FP}$, and JH underestimated MCAP measurements (ES $=-0.1 .00[-1.36 ;-0.63]$ and $1.25[0.85 ; 1.63]$, respectively). Estimated PP obtained from MyJump was largely associated with and not different from PP from MCAP $(r=0.61[0.46 ; 0.72] ; E S=-0.15[-0.49 ;-0.20])$. A huge underestimation was found for MyJump-derived PV compared to MCAP (ES = 9.65 [8.05; 11.06]). Estimated PP assessed via all devices was not valid in relation to FP measurements. 


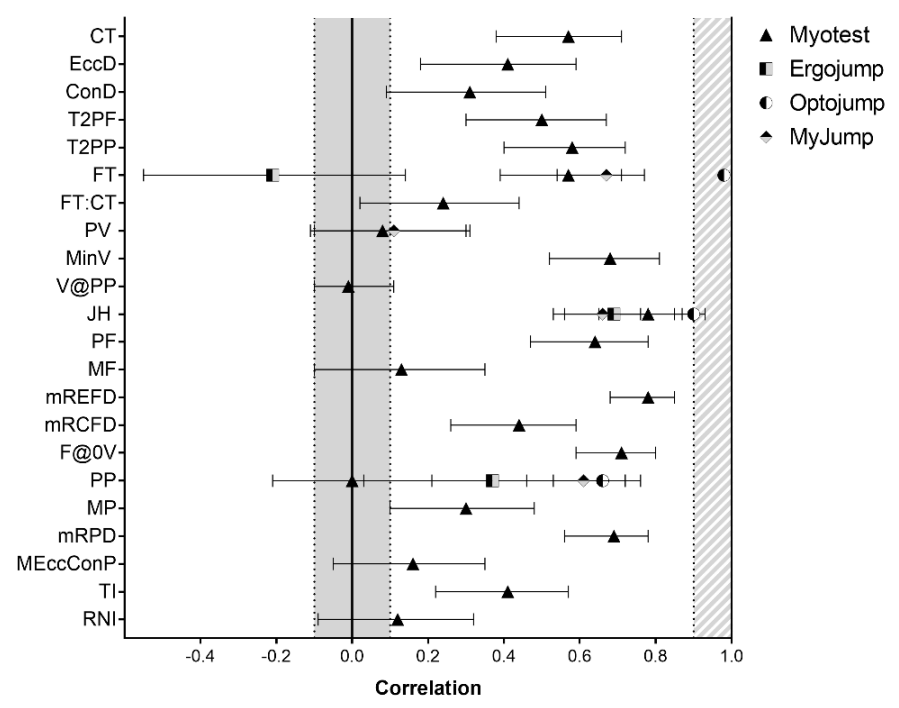

Figure 2. Correlations between gold standard measurements and four portable devices in 22 neuromuscular parameters. Filled gray area indicates no significant correlation $(\mathrm{r}<0.1)$. Shaded space indicates almost perfect correlation $(\mathrm{r} \geq 0.9)$. ConD, concentric duration; $\mathrm{CT}$, total contraction time; EccD, eccentric duration; ES, effect size; F@0V, force at zero velocity; FT, flight time; FT:CT, flight time to contraction time ratio; ICC, intraclass correlation coefficient; $\mathrm{MF}$, mean force; $\mathrm{MP}$, mean power; MEccConP, mean eccentric and concentric power over time; MinV, minimum velocity; mRCFD, maximum rate of concentric force development; mREFD, maximum rate of eccentric force development; mRPD, maximum rate of power development; PF, peak force; PP, peak power; PV, peak velocity; RNI, relative net impulse; T2PF, time to peak force; T2PP, time to peak power; TI, total impulse; V@PP, velocity at peak power. Gold standard is a motion capture system for $\mathrm{PV}, \mathrm{MinV}$, and $\mathrm{JH}$, and force platform for all remaining variables.

A detailed description of test-retest reliability is reported in Table 3. FT and JH were reliable for all portable devices (ICC $=0.54-0.97, \mathrm{CV}=1.8-4.2 \%$ ). $\mathrm{CT}$, EccD, time to peak force (T2PF), time to peak power (T2PP), flight time to contraction time ratio (FT:CT), PF, MF, F@0V, and mean eccentric and concentric power over time (MEccConP) obtained from Myotest showed good absolute and relative reliability (ICC $=0.89-0.97, \mathrm{CV}=3.8-9.5 \%$ ). PV obtained from MyJump also showed good test-retest reliability (ICC [ $95 \% \mathrm{CI}]=0.97$ [0.91; 0.99$] ; \mathrm{CV}=3.9 \%$ ). With the exception of Myotest, $\mathrm{PP}$ was reliable for all portable devices (ICC $=0.84-0.91, C V=3.3-4.5 \%$ ). In all devices, all variables showed good capacity to detect changes $(\mathrm{SEM}<\mathrm{SWC})$, except for $\mathrm{mREFD}$, $\mathrm{mRCFD}$, and $\mathrm{mRPD}(\mathrm{SEM}>\mathrm{SWC})($ Table 4$)$. 
Table 3. Test-retest reliability of CMJ kinematic variables obtained with two gold-standard pieces of equipment and four portable devices.

\begin{tabular}{|c|c|c|c|c|c|c|c|}
\hline Variable & $\begin{array}{l}\text { Reliability } \\
\text { Indicators }\end{array}$ & MCAP & Force Platform & Myotest & Ergojump & Optojump & MyJump \\
\hline \multirow{2}{*}{$\mathrm{CT}$} & $\operatorname{ICC}(95 \% \mathrm{CI})$ & - & $0.84(0.58 ; 0.95)$ & $0.93(0.83 ; 0.98)$ & - & - & - \\
\hline & CV $(\%)$ & - & $120.1(7.7 ; 16.5)$ & $6.8(4.9 ; 8.7)$ & - & - & - \\
\hline \multirow{2}{*}{ EccD } & ICC $(95 \% \mathrm{CI})$ & - & $0.81(0.51 ; 0.94)$ & $0.95(0.87 ; 0.98)$ & - & - & - \\
\hline & CV $(\%)$ & - & $17.5(10.1 ; 24.9)$ & $8.0(5.7 ; 10.3)$ & - & - & - \\
\hline \multirow{2}{*}{ ConD } & ICC $(95 \%$ CI $)$ & - & $0.89(0.71 ; 0.96)$ & $0.72(0.27 ; 0.92)$ & - & - & - \\
\hline & CV $(\%)$ & - & $6.8(5.2 ; 8.3)$ & $12.0(7.1 ; 16.9)$ & - & - & - \\
\hline \multirow{2}{*}{$\mathrm{T} 2 \mathrm{PF}$} & ICC $(95 \% \mathrm{CI})$ & - & $0.80(0.48 ; 0.94)$ & $0.93(0.82 ; 0.98)$ & - & - & - \\
\hline & CV $(\%)$ & - & $21.1(14.4 ; 27.7)$ & $7.9(5.8 ; 10.1)$ & - & - & - \\
\hline \multirow{2}{*}{$\mathrm{T} 2 \mathrm{PP}$} & $\operatorname{ICC}(95 \% \mathrm{CI})$ & - & $0.82(0.52 ; 0.95)$ & $0.93(0.83 ; 0.98)$ & - & - & - \\
\hline & CV $(\%)$ & - & $13.3(8.6 ; 18.1)$ & $7.0(5.1 ; 8.9)$ & - & - & - \\
\hline \multirow{2}{*}{ FT } & $\operatorname{ICC}(95 \% \mathrm{CI})$ & - & $0.97(0.92 ; 0.99)$ & $0.96(0.91 ; 0.99)$ & $0.54(-0.34 ; 0.89)$ & $0.96(0.91 ; 0.99)$ & $0.80(0.37 ; 0.96)$ \\
\hline & CV $(\%)$ & - & $1.8(1.3 ; 2.2)$ & $3.8(0.4 ; 7.2)$ & $8.2(0.8 ; 15.7)$ & $1.8(1.4 ; 2.3)$ & $3.0(2.1 ; 3.8)$ \\
\hline \multirow{2}{*}{ FT:CT } & $\operatorname{ICC}(95 \% \mathrm{CI})$ & - & $0.73(0.30 ; 0.92)$ & $0.92(0.78 ; 0.98)$ & - & - & - \\
\hline & CV $(\%)$ & - & $13.2(8.0 ; 18.3)$ & $8.4(3.4 ; 13.4)$ & - & - & - \\
\hline \multirow{2}{*}{ PV } & $\operatorname{ICC}(95 \% \mathrm{CI})$ & $0.91(0.78 ; 0.97)$ & - & $0.78(0.44 ; 0.94)$ & - & - & $0.74(0.17 ; 0.95)$ \\
\hline & CV $(\%)$ & $5.2(3.2 ; 7.1)$ & - & $11.6(7.6 ; 15.3)$ & - & - & $3.3(2.2 ; 4.4)$ \\
\hline \multirow{2}{*}{ MinV } & $\operatorname{ICC}(95 \% \mathrm{CI})$ & $0.79(-39 ; 0.95)$ & - & $0.50(-0.30 ; 0.86)$ & - & - & - \\
\hline & CV $(\%)$ & $-6.8(-8.0 ;-5.6)$ & - & $-18.0(-24.6 ;-12.3)$ & - & - & - \\
\hline \multirow{2}{*}{ V@PP } & ICC $(95 \% \mathrm{CI})$ & - & $0.97(0.92 ; 0.99)$ & $0.79(0.46 ; 0.94)$ & - & - & - \\
\hline & CV $(\%)$ & - & $2.1(1.54 ; 2.6)$ & $11.1(7.7 ; 14.5)$ & - & - & - \\
\hline \multirow{2}{*}{$\mathrm{JH}$} & $\operatorname{ICC}(95 \% \mathrm{CI})$ & $0.96(0.90 ; 0.99)$ & - & $0.97(0.92 ; 0.99)$ & $0.93(0.81 ; 0.98)$ & $0.87(0.62 ; 0.97)$ & $0.97(0.91 ; 0.99)$ \\
\hline & CV $(\%)$ & $3.3(2.2 ; 4.4)$ & - & $4.2(3.2 ; 5.3)$ & $3.2(2.3 ; 4.1)$ & $4.2(3.3 ; 5.1)$ & $3.9(3.0 ; 4.9)$ \\
\hline \multirow{2}{*}{ PF } & $\operatorname{ICC}(95 \% \mathrm{CI})$ & - & $0.83(0.57 ; 0.95)$ & $0.91(0.77 ; 0.97)$ & - & - & - \\
\hline & CV $(\%)$ & - & $3.4(2.4 ; 4.5)$ & $6.4(4.4 ; 8.3)$ & - & - & - \\
\hline \multirow{2}{*}{ MF } & $\operatorname{ICC}(95 \% \mathrm{CI})$ & - & $0.89(-0.73 ; 0.96)$ & $0.89(0.70 ; 0.96)$ & - & - & - \\
\hline & CV $(\%)$ & - & $5.2(1.9 ; 8.5)$ & $4.2(2.5 ; 5.9)$ & - & - & - \\
\hline \multirow{2}{*}{ mREFD } & $\operatorname{ICC}(95 \% \mathrm{CI})$ & - & $0.89(0.71 ; 0.97)$ & $0.76(0.37 ; 0.93)$ & - & - & - \\
\hline & CV $(\%)$ & - & $17.1(10.8 ; 23.4)$ & $21.1(15.3 ; 26.8)$ & - & - & - \\
\hline
\end{tabular}


Table 3. Cont.

\begin{tabular}{|c|c|c|c|c|c|c|c|}
\hline Variable & $\begin{array}{l}\text { Reliability } \\
\text { Indicators }\end{array}$ & MCAP & Force Platform & Myotest & Ergojump & Optojump & MyJump \\
\hline \multirow{3}{*}{ mRCFD } & $\operatorname{ICC}(95 \% \mathrm{CI})$ & - & $0.84(0.62 ; 0.95)$ & $0.28(-0.29 ; 0.86)$ & - & - & - \\
\hline & $\mathrm{CV}(\%)$ & - & $46.4(30.4 ; 62.4)$ & $30.9(20.9 ; 40.9)$ & - & - & - \\
\hline & F@0V & - & - & - & - & - & - \\
\hline \multirow{2}{*}{ F@0V } & $\operatorname{ICC}(95 \% \mathrm{CI})$ & - & $0.90(0.74 ; 0.97)$ & $0.91(0.77 ; 0.97)$ & - & - & - \\
\hline & CV $(\%)$ & - & $4.3(2.7 ; 5.9)$ & $8.9(5.6 ; 12.2)$ & - & - & - \\
\hline \multirow{2}{*}{$\mathrm{PP}$} & $\operatorname{ICC}(95 \% \mathrm{CI})$ & - & $0.95(0.87 ; 0.98)$ & $0.81(0.52 ; 0.94)$ & $0.91(0.79 ; 0.97)$ & $0.86(0.63 ; 0.96)$ & $0.84(0.48 ; 0.96)$ \\
\hline & CV $(\%)$ & - & $3.4(2.1 ; 4.7)$ & $15.8(11.3 ; 20.4)$ & $3.3(2.3 ; 4.3)$ & $4.5(1.0 ; 8.1)$ & $4.0(3.0 ; 5.0)$ \\
\hline \multirow{3}{*}{$\mathrm{MP}$} & $\operatorname{ICC}(95 \%$ CI $)$ & - & $0.91(0.77 ; 0.97)$ & $0.84(0.59 ; 0.95)$ & - & - & - \\
\hline & CV $(\%)$ & - & $3.8(2.7 ; 5.0)$ & $14.8(10.2 ; 19.3)$ & - & - & - \\
\hline & $m R P D$ & - & - & - & - & - & - \\
\hline \multirow{2}{*}{$\mathrm{mRPD}$} & $\operatorname{ICC}(95 \% \mathrm{CI})$ & - & $0.75(0.34 ; 0.93)$ & $0.84(0.58 ; 0.95)$ & - & - & - \\
\hline & CV $(\%)$ & - & $10.1(8.0 ; 12.3)$ & $16.0(10.6 ; 21.3)$ & - & - & - \\
\hline \multirow{2}{*}{ MEccCONP } & $\operatorname{ICC}(95 \% \mathrm{CI})$ & - & $0.87(0.65 ; 0.96)$ & $0.93(0.81 ; 0.98)$ & - & - & - \\
\hline & CV $(\%)$ & - & $9.0(5.9 ; 12.1)$ & $9.5(5.8 ; 13.2)$ & - & - & - \\
\hline \multirow{2}{*}{ TI } & $\operatorname{ICC}(95 \% \mathrm{CI})$ & - & $0.79(0.44 ; 0.94)$ & $0.95(0.87 ; 0.98)$ & - & - & - \\
\hline & CV $(\%)$ & - & $5.6(1.3 ; 9.9)$ & $24.4(10.8 ; 37.9)$ & - & - & - \\
\hline \multirow{2}{*}{ RNI } & $\operatorname{ICC}(95 \% \mathrm{CI})$ & - & $0.97(0.92 ; 0.99)$ & $0.86(0.63 ; 0.96)$ & - & - & - \\
\hline & CV $(\%)$ & - & $1.7(1.1 ; 2.2)$ & $15.9(10.1 ; 21.8)$ & - & - & - \\
\hline
\end{tabular}

MCAP, motion capture system; ConD, concentric duration; CT, total contraction time; EccD, eccentric duration; F@0V, force at zero velocity; FT, flight time; FT:CT, flight time to contraction time ratio; $\mathrm{MP}$, mean power; $\mathrm{MEccConP}$, mean eccentric and concentric power over time; MinV, minimum velocity; mRCFD, maximum rate of concentric force development; mRCED, maximum rate of eccentric force development; $\mathrm{mRPD}$, maximum rate of power development; PF, peak force; PP, peak power; PV, peak velocity; RNI, relative net impulse; T2PF, time to peak force; T2PP, time to peak power; TI, total impulse; V@PP, velocity at peak power. Bold indicates reliable measurements (ICC $>0.75, \mathrm{CV}<10 \%$ ). 
Table 4. Standard error of measurement, smallest worthwhile change, and minimum detectable change of jump variables obtained with different devices.

\begin{tabular}{|c|c|c|c|c|c|c|c|c|c|c|c|c|c|c|c|c|c|c|}
\hline \multirow[t]{2}{*}{ Variable } & \multicolumn{3}{|c|}{ MCAP } & \multicolumn{3}{|c|}{ Force Platform } & \multicolumn{3}{|c|}{ Myotest } & \multicolumn{3}{|c|}{ Ergojump } & \multicolumn{3}{|c|}{ Optojump } & \multicolumn{3}{|c|}{ MyJump } \\
\hline & SEM & SWC & MDC & SEM & SWC & MDC & SEM & SWC & MDC & SEM & SWC & MDC & SEM & SWC & MDC & SEM & SWC & MDC \\
\hline \multicolumn{19}{|c|}{ Kinematic parameters } \\
\hline CT (s) & - & - & - & 0.02 & 0.03 & 0.05 & 0.012 & 0.018 & 0.033 & - & - & - & - & - & - & - & - & - \\
\hline $\operatorname{EccD}(\mathrm{s})$ & - & - & - & 0.01 & 0.03 & 0.05 & 0.010 & 0.016 & 0.029 & - & - & - & - & - & - & - & - & - \\
\hline ConD (s) & - & - & - & 0.003 & 0.006 & 0.01 & 0.005 & 0.008 & 0.015 & - & - & - & - & - & - & - & - & - \\
\hline T2PF (s) & - & - & - & 0.02 & 0.03 & 0.06 & 0.013 & 0.021 & 0.038 & - & - & - & - & - & - & - & - & - \\
\hline $\mathrm{T} 2 \mathrm{PP}(\mathrm{s})$ & - & - & - & 0.02 & 0.03 & 0.06 & 0.011 & 0.018 & 0.033 & - & - & - & - & - & - & - & - & - \\
\hline FT (s) & - & - & - & 0.004 & 0.006 & 0.01 & 0.005 & 0.008 & 0.015 & 0.008 & 0.013 & 0.024 & 0.004 & 0.006 & 0.012 & 0.003 & 0.006 & 0.010 \\
\hline FT:CT (\%) & - & - & - & 0.02 & 0.03 & 0.05 & 0.013 & 0.021 & 0.038 & - & & - & - & - & - & - & - & - \\
\hline $\mathrm{PV}\left(\mathrm{m} \cdot \mathrm{s}^{-1}\right)$ & 0.04 & 0.06 & 0.11 & - & - & - & 0.050 & 0.078 & 0.14 & - & & - & - & - & - & - & - & - \\
\hline $\operatorname{MinV}\left(\mathrm{m} \cdot \mathrm{s}^{-1}\right)$ & 0.02 & 0.03 & 0.05 & - & - & - & 0.032 & 0.050 & 0.090 & - & & - & - & - & - & - & - & - \\
\hline $\mathrm{V} @ P P\left(\mathrm{~m} \cdot \mathrm{s}^{-1}\right)$ & - & - & - & 0.02 & 0.03 & 0.05 & 0.04 & 0.06 & 0.056 & - & & - & - & - & - & 0.009 & 0.015 & 0.027 \\
\hline $\mathrm{JH}(\mathrm{m})$ & 0.005 & 0.008 & 0.015 & - & - & - & 0.005 & 0.008 & 0.014 & 0.010 & 0.015 & 0.028 & 0.005 & 0.009 & 0.016 & 0.005 & 0.008 & 0.015 \\
\hline \multicolumn{19}{|c|}{ Kinetic parameters } \\
\hline $\mathrm{PF}\left(\mathrm{N} \cdot \mathrm{kg}^{-1}\right)$ & - & - & - & 0.26 & 0.40 & 0.73 & 0.30 & 0.47 & 0.84 & - & - & - & - & - & - & - & - & - \\
\hline $\mathrm{MF}\left(\mathrm{N} \cdot \mathrm{kg}^{-1}\right)$ & - & - & - & 0.16 & 0.26 & 0.47 & 0.10 & 0.16 & 0.47 & - & - & - & - & - & - & - & - & - \\
\hline $\operatorname{mREFD}\left(\mathrm{N} \cdot \mathrm{s}^{-1} \cdot \mathrm{kg}^{-1}\right)$ & - & - & - & 9.42 & 1.88 & 26.11 & 4.88 & 0.97 & 13.53 & - & - & - & - & - & - & - & - & - \\
\hline $\mathrm{mRCFD}\left(\mathrm{N} \cdot \mathrm{s}^{-1} \cdot \mathrm{kg}^{-1}\right)$ & - & - & - & 2.74 & 0.54 & 7.59 & 3.46 & 0.69 & 9.61 & - & - & - & - & - & - & - & - & - \\
\hline $\mathrm{F} @ 0 \mathrm{~V}\left(\mathrm{~N} \cdot \mathrm{kg}^{-1}\right)$ & - & - & - & 0.30 & 0.47 & 0.84 & 0.42 & 0.65 & 1.17 & - & - & - & - & - & - & - & - & - \\
\hline $\mathrm{PP}\left(\mathrm{W} \cdot \mathrm{kg}^{-1}\right)$ & - & - & - & 0.63 & 0.98 & 1.76 & 1.13 & 1.76 & 3.15 & 1.31 & 2.03 & 3.63 & 0.69 & 1.07 & 1.92 & 0.49 & 0.76 & 1.36 \\
\hline $\mathrm{MP}\left(\mathrm{W} \cdot \mathrm{kg}^{-1}\right)$ & - & - & - & 0.32 & 0.51 & 0.91 & 0.67 & 1.03 & 1.86 & - & - & - & - & - & - & - & - & - \\
\hline $\operatorname{mRPD}\left(\mathrm{W} \cdot \mathrm{s}^{-1}\right)$ & - & - & - & 11.38 & 2.27 & 31.54 & 0.30 & 0.06 & 0.84 & - & - & - & - & - & - & - & - & - \\
\hline $\begin{array}{c}\text { MEccConP } \\
\left(\mathrm{W} \cdot \mathrm{kg}^{-1} \cdot \mathrm{s}^{-1}\right)\end{array}$ & - & - & - & 0.29 & 0.45 & 0.80 & 0.25 & 0.40 & 0.71 & - & - & - & - & - & - & - & - & - \\
\hline TI $\left(\mathrm{N} \cdot \mathrm{s}^{-1}\right)$ & - & - & - & 4.06 & 6.29 & 11.26 & 6.24 & 9.67 & 17.30 & - & - & - & - & - & - & - & - & - \\
\hline $\mathrm{RNI}\left(\mathrm{N} \cdot \mathrm{s}^{-1} \cdot \mathrm{kg}^{-1}\right)$ & - & - & - & 0.02 & 0.03 & 0.05 & 0.05 & 0.08 & 0.14 & - & - & - & - & - & - & - & - & - \\
\hline
\end{tabular}

$\mathrm{MCAP}$, motion capture system; ConD, concentric duration; CT, total contraction time; EccD, eccentric duration; F@0V, force at zero velocity; FT, flight time; FT:CT, flight time to contraction time ratio; $\mathrm{MCD}$, minimal detectable change; $\mathrm{MF}$, mean force; $\mathrm{MP}$, mean power; $\mathrm{MEccConP}$, mean eccentric and concentric power over time; $\mathrm{MEF}$, mean eccentric force; MinV, minimum velocity; $\mathrm{mRCFD}$, maximum rate of concentric force development; $\mathrm{mRCED}$, maximum rate of eccentric force development; $\mathrm{mRPD}$, maximum rate of power development; $\mathrm{PF}$, peak force; $\mathrm{PP}$, peak power; $\mathrm{PV}$, peak velocity; RNI, relative net impulse; SEM, standard error of measurement; $\mathrm{SWC}$, smallest worthwhile change; T2PF, time to peak force; T2PP, time to peak power; TI, total impulse; V@PP, velocity at peak power. 


\section{Discussion}

To best of our knowledge, this is the first study assessing the validity and reliability of different portable devices to measure CMJ-derived parameters. Different instruments may be used in the field according to the targeted neuromuscular parameter. Specifically, Myotest is valuable for measuring CMJ-derived temporal parameters during ground contact (e.g., CT and EccD), whereas Ergojump, Optojump, and MyJump may be preferable for measuring FT and JH. On the other hand, FT obtained from Optojump was the only variable that was either valid (in relation to gold-standard devices) and reliable (test-retest) to measure CMJ performance. FT should be used as the criterion measure of jump performance instead of $\mathrm{JH}$, since it is directly measured. Indeed, $\mathrm{JH}$ is indirectly obtained from an equation that uses the flight time squared [10], which means that the measurement error increases with longer flight times. It should also be noted that the equipment tested for reliability and validity showed good sensitivity to detecting changes in all variables measured, with the exception of mREFD, mRCFD, and mRPD.

In this study, we used a within-subject design to avoid interjump variability. It has been suggested that method agreement-type studies should involve at least 40 participants for adequate statistical accuracy [25]. Despite our smaller number of participants, repeated measurements on individual subjects (four jumps per subject) resulted in 60 cases, increasing statistical accuracy [26].

Despite the wide use of portable devices, only a few independent research studies have addressed their reliability and validity $[13,14,17,18,27]$. One interesting finding of this research is the validity and reliability of some CMJ-derived parameters (CT, EccD, ConD, PF) obtained from Myotest, possibly of interest for fatigue-monitoring purposes. Gathercole et al. [12] reported small changes in EccD (increase) and PF (decrease) immediately after exercise (three yo-yo intermittent endurance tests separated by $5 \mathrm{~min}$ active recovery), possibly indicating good capacity of these parameters to detect neuromuscular fatigue. Similarly, our study showed that Myotest-derived CT, EccD, ConD, and PF can be used to track changes in neuromuscular function throughout recovery. In addition, reports in professional soccer players have shown that fluctuations in high-intensity running $\left(>14.4 \mathrm{~km} \cdot \mathrm{h}^{-1}\right)$ on the previous day were associated with CMJ-derived JH fluctuations [3]. However, this correlation was small $(r=0.23)$, and therefore we speculate that other neuromuscular parameters may be more sensitive for detecting neuromuscular fatigue induced by training load, rather than JH. Therefore, future studies should employ Myotest in the field on a daily basis and analyze with CMJ-derived variables to investigate fatigue response to training.

It should also be noted that FT, JH, F@0V, FTTPF, TTPP, and FT:CT showed good test-retest reliability in all portable devices, but significant differences from FP measurements. This possibly indicates that these parameters are less accurate for assessing training adaptations and monitoring fatigue. Our results on FT and JH as CMJ performance indicators corroborates results of previous attempts investigating the concurrent validity of Myotest in relation to FP [13] or even to Optojump when assumed as gold standard [14]. Specifically, our study showed that JH evaluated in Myotest was overestimated in Optojump by $\sim 12 \mathrm{~cm}$. This was also observed in previous efforts to concurrently validate assessment of Myotest, employing indirect estimations of JH (calculated from vertical take-off velocity) [14]. However, when employing the FT equation instead of taking CMJ data from the Myotest display (based on take-off velocity), JH can be more accurately estimated [10]_ENREF_9. Indeed, our results show that FT measured by Myotest is neither valid nor reliable. This could partially be attributed to the different methodologies adopted. In the present study, different methods were adopted using direct measurement of JH and FT (by MCAP), possibly obtaining more accurate results than when using a portable device as the gold standard (e.g., Optojump) [14].

Although the results obtained from Ergojump showed a lack of concurrent validity in relation to gold-standard devices $(r=0.61 ;[<0.90])$ it accomplished the reliability assumptions (ICC $=0.54[>0.50]$; $\mathrm{CV}=8.2[<10 \%])$. This is likely because the switch mat directly computes FT using the time taken from take-off to landing. On the other hand, the system indirectly calculates JH based on flight time, possibly 
explaining observed differences $(<8 \mathrm{~cm})$ compared to the MCAP. Future studies may implement a more rigorous protocol with more jump trials, which could contribute to increased reliability.

Sharing the same method of calculation, JH outcomes from Optojump and Ergojump are similar, except for validity ( $r=0.98$ and 0.61 , respectively). Indeed, FT obtained from Optojump was almost perfectly correlated, reliable, and not different compared to FP. This is in accordance with Castagna et al. [13], who observed a nearly perfect correlation $(r=0.99)$ and no significant differences between FT measured in Optojump and FP. Moreover, Glatthorn et al. [16] reported a correlation coefficient of 0.99 between Optojump and FP for JH values, even if a systematic bias of $\sim 1 \mathrm{~cm}$ was observed. In addition, previous reports showed excellent test-retest reliability (ICC $=0.98, \mathrm{CV}=2.7 \%$ ) of JH from Optojump and low random error between repetitions $(\sim 2.8 \mathrm{~cm})$ [16].

In the present study, despite large association and good reliability, FT and JH obtained from MyJump were significantly different from gold-standard devices. JH findings are partially in agreement with previous reports showing either significant associations $(r=0.96-0.99)$ or no differences between MyJump and FP $[17,28]$. In this study, differences in JH between MyJump and gold-standard devices could be attributed to the fact that the take-off and landing frame selection in MyJump was performed manually, which could increase the measurement error. Fortunately, the continued progress of technology indicates that in the near future smartphones will include cameras with higher recording frequencies, which may reduce the measurement error of MyJump.

The rate of force and rate of power development indicate how rapidly force or power changes (i.e., rate of change) from the beginning to the end of the CMJ. Reliability statistics showed that mRCFD, $\mathrm{mRCED}$, and $\mathrm{mRPD}$ have larger variability than the other variables. These findings are supported by previous research investigating the test-retest reliability of $\mathrm{mRFD}(\mathrm{CV}=16-35 \%)$ [20]. This inherent variability may be explained by dynamic system theory, which suggests that movement coordination is adjusted for perturbation to obtain a consistent performance outcome, and therefore both the rate of force and the rate of power development during CMJ may be more reflective of coordination adjustment than the characteristics of the lower extremities [20].

As a main limitation, the subjects differed in sports activities (e.g., weight lifting, resistance training, and recreational team sports), possibly affecting our results. Therefore, future studies should explore the reliability of these parameters in professional athletes.

Athletes are subject to frequent alterations to their training schedule, according to coaches' decisions and the competitive calendar. Therefore, another important aspect of field testing is the flexibility allowed by the coach to change assessment scheduling, without any commitment to external laboratories. We used the standardized CMJ version (hands remained in contact with the spine of the pelvis) to analyze test-retest reliability. Future studies may use the arm-swing CMJ version to verify whether the jump technique affects the reliability of variables, as well as further novel systems (e.g., Xbox Kinect).

\section{Conclusions}

This study compares a wide range of CMJ-derived variables over six different devices, aiming to analyze the most convenient tool to be employed for on-field neuromuscular testing. Although the portable devices showed different levels of accuracy to measure CMJ according to the specific neuromuscular parameters analyzed, Myotest should be preferred to measure CMJ-derived temporal parameters during ground contact (e.g., CT and EccD). Ergojump, Optojump, and MyJump quantify the time taken from jump take-off to landing and may be adopted to measure FT or JH. Optojump seems to show the most accurate CMJ performance measurement across these devices. Finally, with the exception of rate of force and rate of power development, all parameters analyzed in the current study showed good capacity to detect changes irrespective of the equipment used. The application of portable devices could be of interest for coaches and practitioners to schedule and change testing or training sessions according to the team's or player's calendar, without any commitment to an external laboratory. 
Author Contributions: Conceptualization, V.R., J.B., P.F. (Pedro Figueiredo) and A.R.; Methodology, V.R., J.B., P.F. (Pedro Figueiredo), P.F. (Pedro Fonseca) and A.R.; Software, V.R. and P.F. (Pedro Fonseca); Validation, P.F. (Pedro Fonseca) and A.R.; Formal Analysis, V.R., J.B., P.F. (Pedro Figueiredo), P.F. (Pedro Fonseca) and A.R.; Investigation, V.R., J.B., P.F. (Pedro Figueiredo), P.F. (Pedro Fonseca) and A.R.; Resources, P.F. (Pedro Fonseca); Data Curation, V.R., T.C., T.F. and P.F. (Pedro Fonseca); Writing-Original Draft Preparation, V.R.; Writing-Review \& Editing, V.R., J.B., P.F. (Pedro Figueiredo), T.F., P.F. (Pedro Fonseca) and A.R.; Supervision, P.F. (Pedro Fonseca) and A.R.; Project Administration, V.R., P.F. (Pedro Fonseca) and A.R.

Funding: This research was funded by Fundação para a ciência e tecnologia grant number SFRH/BD/129324/2017 and The APC was funded by Federação Portuguesa de Futebol.

Conflicts of Interest: The authors declare no conflict of interest.

\section{References}

1. Vanezis, A.; Lees, A. A biomechanical analysis of good and poor performers of the vertical jump. Ergonomics 2005, 48, 1594-1603. [CrossRef] [PubMed]

2. Silva, J.R.; Magalhaes, J.F.; Ascensao, A.A.; Oliveira, E.M.; Seabra, A.F.; Rebelo, A.N. Individual match playing time during the season affects fitness-related parameters of male professional soccer players. J. Strength Cond. Res. 2011, 25, 2729-2739. [CrossRef] [PubMed]

3. Thorpe, R.T.; Strudwick, A.J.; Buchheit, M.; Atkinson, G.; Drust, B.; Gregson, W. Monitoring Fatigue During the In-Season Competitive Phase in Elite Soccer Players. Int. J. Sports Physiol. Perform. 2015, 10, 958-964. [CrossRef] [PubMed]

4. Laffaye, G.; Wagner, P.P.; Tombleson, T.I. Countermovement jump height: Gender and sport-specific differences in the force-time variables. J. Strength Cond. Res. 2014, 28, 1096-1105. [CrossRef] [PubMed]

5. Los Arcos, A.; Martinez-Santos, R.; Yanci, J.; Mendiguchia, J.; Mendez-Villanueva, A. Negative Associations between Perceived Training Load, Volume and Changes in Physical Fitness in Professional Soccer Players. J. Sports Sci. Med. 2015, 14, 394-401.

6. Brito, J.; Krustrup, P.; Rebelo, A. The influence of the playing surface on the exercise intensity of small-sided recreational soccer games. Hum. Mov. Sci. 2012, 31, 946-956. [CrossRef] [PubMed]

7. Rebelo, A.N.; Silva, P.; Rago, V.; Barreira, D.; Krustrup, P. Differences in strength and speed demands between 4v4 and 8v8 small-sided football games. J. Sports Sci. 2016, 34, 2246-2254. [CrossRef] [PubMed]

8. Cormack, S.J.; Newton, R.U.; McGuigan, M.R.; Cormie, P. Neuromuscular and endocrine responses of elite players during an Australian rules football season. Int. J. Sports Physiol. Perform. 2008, 3, 439-453. [CrossRef] [PubMed]

9. Gathercole, R.; Sporer, B.; Stellingwerff, T. Countermovement Jump Performance with Increased Training Loads in Elite Female Rugby Athletes. Int. J. Sports Med. 2015, 36, 722-728. [CrossRef] [PubMed]

10. Linthorne, N.P. Analysis of standing vertical jumps using a force platform. Am. J. Phys. 2001, 69, 1198-1204. [CrossRef]

11. Aragón, L.F. Evaluation of Four Vertical Jump Tests: Methodology, Reliability, Validity, and Accuracy. Meas. Phys. Educ. Exerc. Sci. 2000, 4, 215-228. [CrossRef]

12. Gathercole, R.; Sporer, B.; Stellingwerff, T.; Sleivert, G. Alternative countermovement-jump analysis to quantify acute neuromuscular fatigue. Int. J. Sports Physiol. Perform. 2015, 10, 84-92. [CrossRef] [PubMed]

13. Castagna, C.; Ganzetti, M.; Ditroilo, M.; Giovannelli, M.; Rocchetti, A.; Manzi, V. Concurrent validity of vertical jump performance assessment systems. J. Strength Cond. Res. 2013, 27, 761-768. [CrossRef] [PubMed]

14. Casartelli, N.; Muller, R.; Maffiuletti, N.A. Validity and reliability of the Myotest accelerometric system for the assessment of vertical jump height. J. Strength Cond. Res. 2010, 24, 3186-3193. [CrossRef] [PubMed]

15. Bosco, C.; Luhtanen, P.; Komi, P.V. A simple method for measurement of mechanical power in jumping. Eur. J. Appl. Physiol. Occup. Physiol. 1983, 50, 273-282. [CrossRef] [PubMed]

16. Glatthorn, J.F.; Gouge, S.; Nussbaumer, S.; Stauffacher, S.; Impellizzeri, F.M.; Maffiuletti, N.A. Validity and reliability of Optojump photoelectric cells for estimating vertical jump height. J. Strength Cond. Res. 2011, 25, 556-560. [CrossRef] [PubMed]

17. Balsalobre-Fernandez, C.; Glaister, M.; Lockey, R.A. The validity and reliability of an iPhone app for measuring vertical jump performance. J. Sports Sci. 2015, 33, 1574-1579. [CrossRef] [PubMed] 
18. Requena, B.; García, I.; Requena, F.; de Villarreal, E.S.S.; Pääsuke, M. Reliability and Validity of a Wireless Microelectromechanicals Based System (Keimove ${ }^{\mathrm{TM}}$ ) for Measuring Vertical Jumping Performance. J. Sports Sci. Med. 2012, 11, 115-122. [PubMed]

19. Halvorsen, K.; Eriksson, M.; Gullstrand, L.; Tinmark, F.; Nilsson, J. Minimal marker set for center of mass estimation in running. Gait Posture 2009, 30, 552-555. [CrossRef] [PubMed]

20. Mizuguchi, S.; Sands, W.A.; Wassinger, C.A.; Lamont, H.S.; Stone, M.H. A new approach to determining net impulse and identification of its characteristics in countermovement jumping: Reliability and validity. Sports Biomech. 2015, 14, 258-272. [CrossRef] [PubMed]

21. Sayers, S.P.; Harackiewicz, D.V.; Harman, E.A.; Frykman, P.N.; Rosenstein, M.T. Cross-validation of three jump power equations. Med. Sci. Sports Exerc. 1999, 31, 572-577. [CrossRef] [PubMed]

22. Hopkins, W.G.; Marshall, S.W.; Batterham, A.M.; Hanin, J. Progressive statistics for studies in sports medicine and exercise science. Med. Sci. Sports Exerc. 2009, 41, 3-13. [CrossRef] [PubMed]

23. Portney, L.; Watkins, M. Foundations of Clinical Research: Applications to Practice; Pearson/Prentice Hall: Upper Saddle River, NJ, USA, 2015.

24. Weir, J.P. Quantifying test-retest reliability using the intraclass correlation coefficient and the SEM. J. Strength Cond. Res. 2005, 19, 231-240. [PubMed]

25. Hopkins, W.G. Measures of reliability in sports medicine and science. Sports Med. 2000, 30, 1-15. [CrossRef] [PubMed]

26. Atkinson, G.; Davison, R.C.; Nevill, A.M. Performance characteristics of gas analysis systems: What we know and what we need to know. Int. J. Sports Med. 2005, 26 (Suppl. S1), S2-S10. [CrossRef] [PubMed]

27. Choukou, M.A.; Laffaye, G.; Taiar, R. Reliability and validity of an accelerometric system for assessing vertical jumping performance. Biol. Sport 2014, 31, 55-62. [CrossRef] [PubMed]

28. Gallardo-Fuentes, F.; Gallardo-Fuentes, J.; Ramirez-Campillo, R.; Balsalobre-Fernandez, C.; Martinez, C.; Caniuqueo, A.; Canas, R.; Banzer, W.; Loturco, I.; Nakamura, F.Y.; et al. Intersession and Intrasession Reliability and Validity of the My Jump App for Measuring Different Jump Actions in Trained Male and Female Athletes. J. Strength Cond. Res. 2016, 30, 2049-2056. [CrossRef] [PubMed]

(C) 2018 by the authors. Licensee MDPI, Basel, Switzerland. This article is an open access article distributed under the terms and conditions of the Creative Commons Attribution (CC BY) license (http:/ / creativecommons.org/licenses/by/4.0/). 